\title{
On the Optimality of Threshold Policies in Event Triggered Estimation with Packet Drops
}

\author{
Alex S. Leong, Subhrakanti Dey, and Daniel E. Quevedo
}

\begin{abstract}
We consider a remote state estimation problem, where a sensor transmits local state estimates over an independent and identically distributed (i.i.d.) packet dropping link to a remote estimator. At each discrete time instant, the sensor can decide whether to transmit, with each transmission incurring a fixed energy cost. Performance is quantified via an optimization problem that minimizes a convex combination of the expected error covariance at the remote estimator and expected energy usage. For transmission schedules dependent only on the error covariance at the remote estimator, this work establishes that a threshold policy (i.e. transmit if the error covariance exceeds a certain threshold and don't transmit otherwise) is optimal. This provides a rigorous justification for the use of such threshold policies in event triggered estimation. An extension of the result to Markovian packet drops is also outlined.
\end{abstract}

\section{INTRODUCTION}

The concept of event triggered estimation of dynamical systems, where sensor measurements or state estimates are only sent to a remote estimator/controller when certain events occur, has gained recent attention. By transmitting only at events such as when the estimation quality at the remote estimator has deteriorated sufficiently, potential savings in energy usage can be achieved, which are important in networked estimation and control applications. Event triggered estimation has been studied in e.g. [1]-[6], while event triggered control has also been studied in e.g. [7]-[10].

Many rules for deciding when to transmit have been proposed in the literature, such as if the estimation error [1], [2], [4], functions of the estimation error [5], [6], or the error covariance [3], exceeds a given threshold. These transmission policies often lead to energy savings, however the motivation for using these rules are usually based on heuristics. Furthermore, the literature on event triggered estimation is mostly concerned with the idealized case where all scheduled transmissions (when they occur) are received at the remote estimator.

In this paper we allow for the more practical situation where sensor transmissions experience random packet drops [11]. We will focus on transmission schedules which decide whether to transmit based only on knowledge of the error covariance at the remote estimator. Our analysis shows that a threshold policy, where the sensor transmits if the error covariance exceeds a threshold and does not transmit

A. Leong is with the Department of Electrical and Electronic Engineering, University of Melbourne, Parkville, Vic. 3010, Australia. Email: asleong@unimelb.edu.au. S. Dey is with the Department of Engineering Science, Uppsala University, Uppsala, Sweden. E-mail: Subhra.Deyesignal.uu.se. D. Quevedo is with the School of Electrical Engineering and Computer Science, University of Newcastle, Callaghan, NSW 2308, Australia. E-mail: d.quevedo@ieee.org.

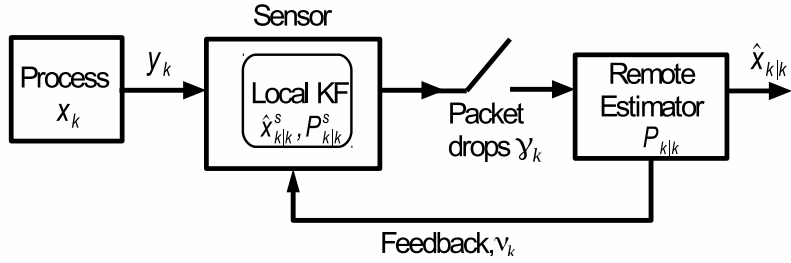

Fig. 1. System model

otherwise, is optimal in the sense that it minimizes a convex combination of the expected error covariance and expected energy usage. This provides a theoretical justification for the use of such threshold policies in event triggered estimation.

The paper is organized as follows. Section II presents the system model, while the optimization problems for the finite and infinite horizon cases are formulated in Section III. The proof that threshold policies are optimal for these optimization problems is given in Section IV. Analytical expressions for the expected energy usage and expected error covariance are derived in Section V. Some numerical results are presented in Section VI. An extension of the main result to Markovian packet drops is outlined in Section VII.

\section{SYSTEM MODEL}

A diagram of the system model is shown in Fig. 1. Consider a discrete time process

$$
x_{k+1}=A x_{k}+w_{k}
$$

with $A$ being unstable, where $x_{k} \in \mathbb{R}^{n}$ and $w_{k}$ is i.i.d. Gaussian with zero mean and covariance $Q$. There is a sensor taking measurements

$$
y_{k}=C x_{k}+v_{k}
$$

where $y_{k} \in \mathbb{R}^{m}$ and $v_{k}$ is i.i.d. Gaussian with zero mean and covariance $R$. We assume that $\left\{w_{k}\right\}$ and $\left\{v_{k}\right\}$ are mutually indepedent, the pair $(A, C)$ is detectable and the pair $\left(A, Q^{1 / 2}\right)$ is stabilizable.

The sensor has computational capability and can run a local Kalman filter. The local state estimates and error covariances

$$
\begin{aligned}
\hat{x}_{k \mid k-1}^{s} & \triangleq \mathbb{E}\left[x_{k} \mid y_{0}, \ldots, y_{k-1}\right] \\
\hat{x}_{k \mid k}^{s} & \triangleq \mathbb{E}\left[x_{k} \mid y_{0}, \ldots, y_{k}\right] \\
P_{k \mid k-1}^{s} & \triangleq \mathbb{E}\left[\left(x_{k}-\hat{x}_{k \mid k-1}^{s}\right)\left(x_{k}-\hat{x}_{k \mid k-1}^{s}\right)^{T} \mid y_{0}, \ldots, y_{k-1}\right] \\
P_{k \mid k}^{s} & \triangleq \mathbb{E}\left[\left(x_{k}-\hat{x}_{k \mid k}^{s}\right)\left(x_{k}-\hat{x}_{k \mid k}^{s}\right)^{T} \mid y_{0}, \ldots, y_{k}\right]
\end{aligned}
$$


can be computed using the local Kalman filter. Let $\bar{P}^{s}$ be the steady state value of $P_{k \mid k-1}^{s}$ and $\bar{P}$ the steady state value of $P_{k \mid k}^{s}$ as $k \rightarrow \infty$, which both exist due to the detectability assumption. To simplify the presentation, we will assume that the local Kalman filter is operating in steady state, so that $P_{k \mid k-1}^{s}=\bar{P}^{s}$ and $P_{k \mid k}^{s}=\bar{P}, \forall k$.

At time $k$, the remote estimator decides whether the sensor should send its state estimates $\hat{x}_{k \mid k}^{s}$. Let $\nu_{k} \in\{0,1\}$ be a decision variable such that $\nu_{k}=1$ if $\hat{x}_{k \mid k}^{s}$ is transmitted to the remote estimator, and $\nu_{k}=0$ if there is no transmission. We will assume that $\nu_{k}$ is communicated by the remote estimator ${ }^{1}$ back to the sensor without error ${ }^{2}$ via a feedback link, see Fig. 1. The decision $\nu_{k}$ is assumed to not depend on the current value of $x_{k}$ (or functions of $x_{k}$ such as measurements and state estimates). In particular, in this paper we will assume that $\nu_{k}$ depends only on the error covariance at the remote estimator, see Section III.

Sensor transmissions are over a packet dropping link. Let $\gamma_{k}$ be a random variable such that $\gamma_{k}=1$ if the transmission is successfully received by the remote estimator, and $\gamma_{k}=0$ otherwise. We begin our analysis by assuming that $\left\{\gamma_{k}\right\}$ is i.i.d. Bernoulli with

$$
\mathbb{P}\left(\gamma_{k}=1\right)=\lambda, \quad \lambda \in(0,1) .
$$

In Section VII we will outline how to treat correlated packet dropouts. Define the information set available at the remote estimator at time $k$ as:

$$
\mathcal{I}_{k} \triangleq\left\{\nu_{0}, \ldots, \nu_{k}, \nu_{0} \gamma_{0}, \ldots, \nu_{k} \gamma_{k}, \nu_{0} \gamma_{0} \hat{x}_{0 \mid 0}^{s}, \ldots, \nu_{k} \gamma_{k} \hat{x}_{k \mid k}^{s}\right\}
$$

Call the state estimates and error covariances at the remote estimator as:

$$
\begin{aligned}
& \hat{x}_{k \mid k} \triangleq \mathbb{E}\left[x_{k} \mid \mathcal{I}_{k}\right] \\
& P_{k \mid k} \triangleq \mathbb{E}\left[\left(x_{k}-\hat{x}_{k \mid k}\right)\left(x_{k}-\hat{x}_{k \mid k}\right)^{T} \mid \mathcal{I}_{k}\right] .
\end{aligned}
$$

Under the above assumptions on $\nu_{k}$ and $\gamma_{k}$, we have (see e.g. [12])

$$
\begin{aligned}
& \hat{x}_{k \mid k}=\left\{\begin{array}{ccc}
\hat{x}_{k \mid k}^{s}, & \nu_{k} \gamma_{k}=1 \\
A \hat{x}_{k-1 \mid k-1} & , \quad \nu_{k} \gamma_{k}=0
\end{array}\right. \\
& P_{k \mid k}=\left\{\begin{array}{cr}
\bar{P} & \nu_{k} \gamma_{k}=1 \\
A P_{k-1 \mid k-1} A^{T}+Q & , \quad \nu_{k} \gamma_{k}=0 .
\end{array}\right.
\end{aligned}
$$

For our subsequent analysis, we introduce

$$
f(X) \triangleq A X A^{T}+Q
$$

and define the countably infinite set

$$
\mathcal{S} \triangleq\left\{\bar{P}, f(\bar{P}), f^{2}(\bar{P}), \ldots\right\},
$$

where $f^{n}($.$) denotes the n$-fold composition of $f($.$) . Then$ it is clear from (1) that $\mathcal{S}$ consists of all possible values of $P_{k \mid k}$ at the remote estimator.

\footnotetext{
${ }^{1}$ Scheduling can also be done at the sensor if $\gamma_{k-1}$ is fed back from the remote estimator to the sensor.

${ }^{2}$ This models the case where the remote estimator has more resources than the sensor and can transmit on the feedback link with very low probability of error.
}

\section{OPTIMIZATION OF TRANSMISSION SCHEDULING}

In this paper, we will consider transmission policies where $\nu_{k}\left(P_{k-1 \mid k-1}\right)$ depends only on $P_{k-1 \mid k-1}$, similar to [3]. From the way in which the error covariances at the remote estimator are updated, such policies will not depend on $x_{k}$, c.f. [5].

To take into account energy usage, we will assume a fixed transmission energy cost of $E$ for each scheduled transmission (when $\nu_{k}=1$ ). We will consider the following finite horizon (of horizon $K$ ) optimization problem:

$$
\begin{aligned}
& \min _{\left\{\nu_{k}\right\}} \sum_{k=1}^{K} \mathbb{E}\left[\beta \operatorname{tr} P_{k \mid k}+(1-\beta) \nu_{k} E \mid P_{0 \mid 0}, \mathcal{I}_{k}, \nu_{k}\right] \\
& =\min _{\left\{\nu_{k}\right\}} \sum_{k=1}^{K} \mathbb{E}\left[\beta \operatorname{tr} P_{k \mid k}+(1-\beta) \nu_{k} E \mid P_{k-1 \mid k-1}, \nu_{k}\right]
\end{aligned}
$$

for some design parameter $\beta \in(0,1)$. Problem (4) minimizes a convex combination of the trace of the expected error covariance at the remote estimator and the expected transmission energy. We note that

$$
\begin{aligned}
\mathbb{E}\left[\operatorname{tr} P_{k \mid k} \mid P_{k-1 \mid k-1}, \nu_{k}\right]= & \nu_{k}\left[\lambda \operatorname{tr} \bar{P}+(1-\lambda) \operatorname{tr} f\left(P_{k-1 \mid k-1}\right)\right] \\
& +\left(1-\nu_{k}\right) \operatorname{tr} f\left(P_{k-1 \mid k-1}\right) \\
= & \nu_{k} \lambda \operatorname{tr} \bar{P}+\left(1-\nu_{k} \lambda\right) \operatorname{tr} f\left(P_{k-1 \mid k-1}\right)
\end{aligned}
$$

where $f($.$) is defined in (2). Problem (4) can thus be solved$ using the dynamic programming algorithm:

$$
\begin{aligned}
& J_{K+1}\left(P_{K \mid K}\right)=0 \\
& J_{k}\left(P_{k-1 \mid k-1}\right)=\min _{\nu_{k} \in\{0,1\}}\left\{\beta\left[\nu_{k} \lambda \operatorname{tr} \bar{P}+\left(1-\nu_{k} \lambda\right) \operatorname{tr} f\left(P_{k-1 \mid k-1}\right)\right]\right. \\
& \left.\quad+(1-\beta) \nu_{k} E+\mathbb{E}\left[J_{k+1}\left(P_{k \mid k}\right) \mid P_{k-1 \mid k-1}, \nu_{k}\right]\right\} \\
& =\min _{\nu_{k} \in\{0,1\}}\left\{\beta\left[\nu_{k} \lambda \operatorname{tr} \bar{P}+\left(1-\nu_{k} \lambda\right) \operatorname{tr} f\left(P_{k-1 \mid k-1}\right)\right]\right. \\
& \quad+(1-\beta) \nu_{k} E+\nu_{k} \lambda J_{k+1}(\bar{P}) \\
& \left.\quad+\left(1-\nu_{k} \lambda\right) J_{k+1}\left(f\left(P_{k-1 \mid k-1}\right)\right)\right\}, \quad k=K, \ldots, 1 .
\end{aligned}
$$

Note that the finite horizon problem (4) can be solved exactly via explicit enumeration, since for a given initial $P_{0 \mid 0}$, the number of possible values for $P_{k \mid k}, k=1, \ldots, K$, is finite.

We will also consider the infinite horizon problem:

$\min _{\left\{\nu_{k}\right\}} \limsup _{K \rightarrow \infty} \frac{1}{K} \sum_{k=1}^{K} \mathbb{E}\left[\beta \operatorname{tr} P_{k \mid k}+(1-\beta) \nu_{k} E \mid P_{k-1 \mid k-1}, \nu_{k}\right]$

which is a Markov decision process (MDP) based stochastic control problem with $\nu_{k}$ as the "action" and $P_{k-1 \mid k-1}$ as the "state" at time $k$. The Bellman equation for problem (6) is

$$
\begin{array}{r}
\rho+h(P)=\min _{\nu \in\{0,1\}}\{\beta[\nu \lambda \operatorname{tr} \bar{P}+(1-\nu \lambda) \operatorname{tr} f(P)] \\
+(1-\beta) \nu E+\nu \lambda h(\bar{P})+(1-\nu \lambda) h(f(P))\}
\end{array}
$$

where $\rho$ is the optimal average cost per stage and $h($.$) is$ the differential cost or relative value function [13]. For the 
infinite horizon problem (6), existence of solutions can be ensured via the following result:

Lemma 3.1: Let $\lambda>1-\frac{1}{\|A\|^{2}}$, where $\|A\|$ denotes the spectral norm of $A$. Then there exists a constant $\rho$ and a function $h($.$) that satisfies the Bellman equation (7).$

Proof: See Appendix.

Remark 3.2: The condition $\lambda>1-\frac{1}{\|A\|^{2}}$ in Lemma 3.1 is similar to the stability condition of [11].

As a consequence of Lemma 3.1, Problem (6) can be solved using methods such as the relative value iteration algorithm [13]. In computations, since the state space is (countably) infinite, one first truncates the state space to $\mathcal{S}^{N} \triangleq\left\{\bar{P}, f(\bar{P}), f^{2}(\bar{P}), \ldots, f^{N-1}(\bar{P})\right\}$, for some $N \in \mathbb{N}$, and then uses the relative value iteration algorithm to solve the resulting finite state space MDP problem, as follows: For a given $N$, define for $t=0,1,2, \ldots$ the value functions $V_{t}():. \mathcal{S}^{N} \rightarrow \mathbb{R}$ by:

$$
\begin{aligned}
& V_{t+1}(P)=\min _{\nu \in\{0,1\}}\{\beta[\nu \lambda \operatorname{tr} \bar{P}+(1-\nu \lambda) \operatorname{tr} f(P)] \\
& \left.\quad+(1-\beta) \nu E+\nu \lambda V_{t}(\bar{P})+(1-\nu \lambda) V_{t}(f(P))\right\} .
\end{aligned}
$$

Let $P_{f} \in \mathcal{S}^{N}$ be fixed. The relative value iteration algorithm is given by:

$$
\begin{aligned}
& h_{t+1}(P) \triangleq V_{t+1}(P)-V_{t+1}\left(P_{f}\right) \\
& =\min _{\nu \in\{0,1\}}\{\beta[\nu \lambda \operatorname{tr} \bar{P}+(1-\nu \lambda) \operatorname{tr} f(P)] \\
& \left.\quad+(1-\beta) \nu E+\nu \lambda h_{t}(\bar{P})+(1-\nu \lambda) h_{t}(f(P))\right\} \\
& -\min _{\nu_{f} \in\{0,1\}}\left\{\beta\left[\nu_{f} \lambda \operatorname{tr} \bar{P}+\left(1-\nu_{f} \lambda\right) \operatorname{tr} f(P)\right]\right. \\
& \left.\quad+(1-\beta) \nu_{f} E+\nu_{f} \lambda h_{t}(\bar{P})+\left(1-\nu_{f} \lambda\right) h_{t}(f(P))\right\} .
\end{aligned}
$$

As $t \rightarrow \infty$, we have $h_{t}(P) \rightarrow h(P), \forall P \in \mathcal{S}^{N}$, with $h($. satisfying the Bellman equation (7). In practice, the algorithm (8) terminates once the differences $h_{t+1}(P)-h_{t}(P)$ become smaller than a desired level of accuracy $\varepsilon$. One then compares the solutions obtained as $N$ increases to determine an appropriate value of $N$ for trunctation of the state space $\mathcal{S}$, see Chapter 8 of [14] for further details.

\section{Optimality of Threshold Policies}

Solutions to the optimization problems (4) and (6) via dynamic programming or solving MDPs do not provide much insight into the form of the optimal solution. In this subsection we will prove that threshold policies (i.e. transmit if and only if the error covariance exceeds some value) are optimal for problems (4) and (6).

\section{A. Preliminaries}

We first develop some ordering properties which are needed in the proof of the main result in Section IV-B. For symmetric matrices $X$ and $Y$, we say that $X \leq Y$ if $Y-X$ is positive semi-definite. In general, " $\leq$ " only gives a partial ordering on the set of symmetric matrices. However, when restricted to the set $\mathcal{S}$ defined in (3), we have a total ordering, i.e. for any two elements $X, Y \in \mathcal{S}$, one has either $X \leq Y$ or $Y \leq X$.

Lemma 4.1: There is a total ordering on the elements of $\mathcal{S}$ given by

Proof: First note that

$$
\bar{P} \leq f(\bar{P}) \leq f^{2}(\bar{P}) \leq \ldots
$$

$$
f(X) \leq f(Y) \text { for } X \leq Y
$$

which can be easily shown from the definition of $f($.$) in (2).$

We proceed by induction. We have that $f(\bar{P}) \geq \bar{P}$ from e.g. [12]. Now assume that $f^{n}(\bar{P}) \geq f^{n-1}(\bar{P})$. Then

$$
f^{n+1}(\bar{P})=f\left(f^{n}(\bar{P})\right) \geq f\left(f^{n-1}(\bar{P})\right)=f^{n}(\bar{P})
$$

where the inequality comes from (9) and the induction hypothesis. Hence by induction

$$
\bar{P} \leq f(\bar{P}) \leq f^{2}(\bar{P}) \leq \ldots
$$

With Lemma 4.1, we next define on $\mathcal{S} \times\{0,1\}$ the following partial ordering:

$$
\left(P_{1}, \nu_{1}\right) \leq\left(P_{2}, \nu_{2}\right) \text { if } P_{1} \leq P_{2} \text { and } \nu_{1} \leq \nu_{2}
$$

where $P_{1}, P_{2} \in \mathcal{S}, \nu_{1}, \nu_{2} \in\{0,1\}$. Also define the join operation " $\vee$ " on $\mathcal{S} \times\{0,1\}$ (see [15], [16]) by

$$
\left(P_{1}, \nu_{1}\right) \vee\left(P_{2}, \nu_{2}\right) \triangleq\left(\max \left(P_{1}, P_{2}\right), \max \left(\nu_{1}, \nu_{2}\right)\right)
$$

and the meet operation " $\wedge$ " on $\mathcal{S} \times\{0,1\}$ by

$$
\left(P_{2}, \nu_{2}\right) \wedge\left(P_{2}, \nu_{2}\right) \triangleq\left(\min \left(P_{1}, P_{2}\right), \min \left(\nu_{1}, \nu_{2}\right)\right) .
$$

Clearly, $\mathcal{S} \times\{0,1\}$ contains the join and meet of each pair of its elements, thus $\mathcal{S} \times\{0,1\}$ is a lattice with respect to the partial order (10).

Consider functions $g(\cdot, \cdot)$ defined on the lattice $X=$ $\mathcal{S} \times\{0,1\}$ with partial order (10). For lattices which can be written as the product of two chains (or totally ordered sets), we have (see [15]):

Definition 1: A real-valued function $g(\cdot, \cdot)$ defined on the lattice $\mathcal{S} \times\{0,1\}$ is submodular on $\mathcal{S} \times\{0,1\}$ if

$$
g\left(P_{1}, \nu_{1}\right)+g\left(P_{2}, \nu_{2}\right) \leq g\left(P_{1}, \nu_{2}\right)+g\left(P_{2}, \nu_{1}\right)
$$

for all $P_{1}, P_{2} \in \mathcal{S}$ and $\nu_{1}, \nu_{2} \in\{0,1\}$, with $P_{1} \geq P_{2}$ and $\nu_{1} \geq \nu_{2}$.

\section{B. Proof of optimality of threshold policies}

We divide the proof into two stages. First, we have the following:

Lemma 4.2: Let the functions $J_{k}(\cdot): \mathcal{S} \rightarrow \mathbb{R}$ be defined recursively as:

$$
\begin{aligned}
& J_{K+1}(P)=0 \\
& J_{k}(P)=\min _{\nu \in\{0,1\}}\{\beta[\nu \lambda \operatorname{tr} \bar{P}+(1-\nu \lambda) \operatorname{tr} f(P)]+(1-\beta) \nu E \\
& \left.\quad+\nu \lambda J_{k+1}(\bar{P})+(1-\nu \lambda) J_{k+1}(f(P))\right\}, \quad k=K, \ldots, 1 .
\end{aligned}
$$


Then the functions $L_{k}(\cdot, \cdot): \mathcal{S} \times\{0,1\} \rightarrow \mathbb{R}, k=1, \ldots, K$ given by

$$
\begin{aligned}
L_{k}(P, \nu) \triangleq & \beta[\nu \lambda \operatorname{tr} \bar{P}+(1-\nu \lambda) \operatorname{tr} f(P)]+(1-\beta) \nu E \\
& +\nu \lambda J_{k+1}(\bar{P})+(1-\nu \lambda) J_{k+1}(f(P))
\end{aligned}
$$

are submodular on $\mathcal{S} \times\{0,1\}$.

Proof: Define

$$
F(P, \nu) \triangleq \beta \operatorname{tr}[\nu \lambda \bar{P}+(1-\nu \lambda) f(P)]+(1-\beta) \nu E .
$$

By Definition 1, we need to show that for $P_{1} \geq P_{2}, \nu_{1} \geq \nu_{2}$, we have:

$$
\begin{aligned}
& F\left(P_{1}, \nu_{1}\right)+F\left(P_{2}, \nu_{2}\right) \leq F\left(P_{1}, \nu_{2}\right)+F\left(P_{2}, \nu_{1}\right) \\
\Leftrightarrow \operatorname{tr}[ & \left.\nu_{1} \lambda \bar{P}+\left(1-\nu_{1} \lambda\right) f\left(P_{1}\right)\right]+(1-\beta) \nu_{1} E \\
& +\operatorname{tr}\left[\nu_{2} \lambda \bar{P}+\left(1-\nu_{2} \lambda\right) f\left(P_{2}\right)\right]+(1-\beta) \nu_{2} E \\
\leq & \operatorname{tr}\left[\nu_{2} \lambda \bar{P}+\left(1-\nu_{2} \lambda\right) f\left(P_{1}\right)\right]+(1-\beta) \nu_{2} E \\
& +\operatorname{tr}\left[\nu_{1} \lambda \bar{P}+\left(1-\nu_{1} \lambda\right) f\left(P_{2}\right)\right]+(1-\beta) \nu_{1} E \\
\Leftrightarrow & \operatorname{tr}\left[\left(1-\nu_{1} \lambda\right)\left(f\left(P_{1}\right)-f\left(P_{2}\right)\right)\right] \leq \operatorname{tr}\left[\left(1-\nu_{2} \lambda\right)\left(f\left(P_{1}\right)-f\left(P_{2}\right)\right)\right] .
\end{aligned}
$$

The latter expression is true by (9) since $P_{1} \geq P_{2}$ and $\nu_{1} \geq$ $\nu_{2}$. Hence $F(\cdot, \cdot)$ is submodular on $\mathcal{S} \times\{0,1\}$.

Now define

$$
G_{k}(P, \nu) \triangleq \nu \lambda J_{k+1}(\bar{P})+(1-\nu \lambda) J_{k+1}(f(P)) .
$$

To prove that $G_{k}(\cdot, \cdot)$ is submodular on $\mathcal{S} \times\{0,1\}$, we need to show that for $P_{1} \geq P_{2}, \nu_{1} \geq \nu_{2}$,

$$
\begin{aligned}
G_{k}\left(P_{1}, \nu_{1}\right)+G_{k}\left(P_{2}, \nu_{2}\right) \leq G_{k}\left(P_{1}, \nu_{2}\right)+G_{k}\left(P_{2}, \nu_{1}\right) \\
\Leftrightarrow \nu_{1} \lambda J_{k+1}(\bar{P})+\left(1-\nu_{1} \lambda\right) J_{k+1}\left(f\left(P_{1}\right)\right) \\
\quad+\nu_{2} \lambda J_{k+1}(\bar{P})+\left(1-\nu_{2} \lambda\right) J_{k+1}\left(f\left(P_{2}\right)\right) \\
\leq \nu_{2} \lambda J_{k+1}(\bar{P})+\left(1-\nu_{2} \lambda\right) J_{k+1}\left(f\left(P_{1}\right)\right) \\
\quad+\nu_{1} \lambda J_{k+1}(\bar{P})+\left(1-\nu_{1} \lambda\right) J_{k+1}\left(f\left(P_{2}\right)\right) \\
\Leftrightarrow\left(1-\nu_{1} \lambda\right)\left[J_{k+1}\left(f\left(P_{1}\right)\right)-J_{k+1}\left(f\left(P_{2}\right)\right)\right] \\
\leq\left(1-\nu_{2} \lambda\right)\left[J_{k+1}\left(f\left(P_{1}\right)\right)-J_{k+1}\left(f\left(P_{2}\right)\right)\right]
\end{aligned}
$$

By induction, one can show that $J_{k}(P)$ is a non-decreasing function of $P$. Hence the inequality

$$
\begin{aligned}
& \left(1-\nu_{1} \lambda\right)\left[J_{k+1}\left(f\left(P_{1}\right)\right)-J_{k+1}\left(f\left(P_{2}\right)\right)\right] \\
& \quad \leq\left(1-\nu_{2} \lambda\right)\left[J_{k+1}\left(f\left(P_{1}\right)\right)-J_{k+1}\left(f\left(P_{2}\right)\right)\right]
\end{aligned}
$$

is true when $P_{1} \geq P_{2}$ and $\nu_{1} \geq \nu_{2}$, which means that $G_{k}(.,$. is submodular on $\mathcal{S} \times\{0,1\}$.

Since the sum of two submodular functions is submodular,

$$
L_{k}(P, \nu)=F(P, \nu)+G_{k}(P, \nu)
$$

is submodular.

We now give the main technical result of this section:

Theorem 4.3: (i) The optimal solution to the finite horizon problem (4) is of the form:

$$
\nu_{k}^{*}= \begin{cases}0 & , \quad P_{k-1 \mid k-1}<P_{k-1 \mid k-1}^{\mathrm{th}} \\ 1 & , \quad P_{k-1 \mid k-1} \geq P_{k-1 \mid k-1}^{\text {th }}\end{cases}
$$

for some thresholds $P_{k-1 \mid k-1}^{\text {th }} \in \mathcal{S}, k=1, \ldots, K$, where $\mathcal{S}$ is given by (3). (ii) The optimal solution to the infinite horizon problem (6) is of the form:

$$
\nu_{k}^{*}=\left\{\begin{array}{lll}
0 & , \quad P_{k-1 \mid k-1}<P^{\mathrm{th}} \\
1 & , \quad P_{k-1 \mid k-1} \geq P^{\mathrm{th}}
\end{array}\right.
$$

for some constant threshold $P^{\text {th }} \in \mathcal{S}$.

Proof: (i) Lemma 4.2 implies (see [15]) that

$$
\arg \min _{\nu_{k} \in\{0,1\}} L_{k}\left(P_{k-1 \mid k-1}, \nu_{k}\right)
$$

is non-decreasing in $P_{k-1 \mid k-1}$. Since $\nu_{k}$ only takes on one of the two values 0 and 1 , this then means that for each $k \in\{1, \ldots, K\}$ there exists some threshold $P_{k-1 \mid k-1}^{\text {th }} \in \mathcal{S}$ such that $\nu_{k}^{*}=0$ for $P_{k-1 \mid k-1}<P_{k-1 \mid k-1}^{\text {th }}$, and $\nu_{k}^{*}=1$ for $P_{k-1 \mid k-1} \geq P_{k-1 \mid k-1}^{\mathrm{th}}$. This proves the optimality of threshold policies for the finite horizon problem (4).

(ii) For the infinite horizon problem (6), recall the relative value iteration algorithm (8). By similar arguments as in the proof of Lemma 4.2, one can show that the function

$$
\begin{aligned}
& \beta[\nu \lambda \operatorname{tr} \bar{P}+(1-\nu \lambda) \operatorname{tr} f(P)]+(1-\beta) \nu E+\nu \lambda h_{t}(\bar{P}) \\
& +(1-\nu \lambda) h_{t}(f(P))-\min _{\nu_{f} \in\{0,1\}}\left\{\beta\left[\nu_{f} \lambda \operatorname{tr} \bar{P}+\left(1-\nu_{f} \lambda\right) \operatorname{tr} f\left(P_{f}\right)\right]\right. \\
& \left.\quad+(1-\beta) \nu_{f} E+\nu_{f} \lambda h_{t}(\bar{P})+\left(1-\nu_{f} \lambda\right) h_{t}\left(f\left(P_{f}\right)\right)\right\}
\end{aligned}
$$

is submodular, and hence a threshold policy also holds for each $t$. Since $h_{t}(P) \rightarrow h(P)$ as $t \rightarrow \infty$, these thresholds also converge. Thus the optimal policy in the infinite horizon case is $\nu_{k}^{*}=0$ for $P_{k-1 \mid k-1}<P^{\text {th }}$, and $\nu_{k}^{*}=1$ for $P_{k-1 \mid k-1} \geq$ $P^{\text {th }}$, for some constant threshold $P^{\text {th }} \in \mathcal{S}$.

Remark 4.4: In Theorem 4.3, we could have $P_{k-1 \mid k-1}^{\text {th }}$ or $P^{\text {th }}$ equal to $\bar{P}$, in which case $\nu_{k}^{*}=1, \forall P_{k-1 \mid k-1} \in \mathcal{S}$.

Remark 4.5: Knowing that the optimal policy is a threshold policy allows for significant reductions in the amount of computation required to solve problems (4) and (6), see [17] for details.

\section{ANALYTICAL CHARACTERIZATION OF PERFORMANCE}

In the previous subsection we showed that the optimal policy is a threshold policy on the error covariance. This also allows us to derive analytical expressions for the expected energy usage and expected error covariance. We will consider the infinite horizon case here.

Let $t \in \mathbb{N}$ be such that $f^{t}(\bar{P})=P^{\text {th }} \in \mathcal{S}$, see (11). Note that $t$ will depend on the value of $\beta$ chosen in problem (6). Then the evolution of the error covariance at the remote estimator can be modelled as the Markov chain shown in Fig. 2, where state $i$ of the Markov chain corresponds to the value $f^{i}(\bar{P}), i=0,1,2, \ldots$, with $f^{0}(\bar{P}) \triangleq \bar{P}$.

The transition probability matrix $\mathbf{P}$ for the (infinite) Markov chain can be written as:

$\mathbf{P}=\left[\begin{array}{ccccccccc}0 & 1 & 0 & \ldots & \ldots & & & & \cdots \\ 0 & 0 & 1 & 0 & \ldots & & & & \cdots \\ \vdots & & \ddots & \ddots & & & & & \\ 0 & \ldots & \ldots & 0 & 1 & 0 & \ldots & & \ldots \\ \lambda & 0 & \ldots & & 0 & 1-\lambda & 0 & \ldots & \ldots \\ \lambda & 0 & \ldots & & & 0 & 1-\lambda & 0 & \ldots \\ \vdots & \vdots & & & & & & & \ddots\end{array}\right]$. 


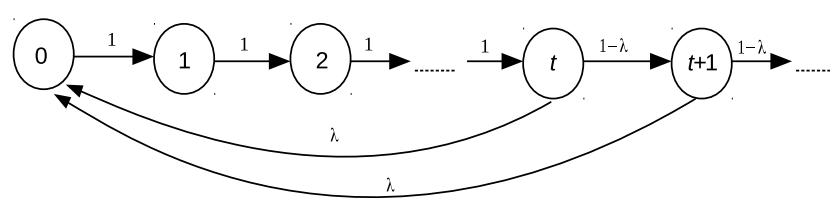

Fig. 2. Markov chain for threshold policy

For $\lambda \in(0,1)$, one can easily verify that the Markov chain is irreducible, aperiodic, and with all states being positive recurrent. Then the stationary distribution

$$
\pi=\left[\begin{array}{llllllll}
\pi_{0} & \pi_{1} & \pi_{2} & \ldots & \pi_{t} & \pi_{t+1} & \pi_{t+2} & \ldots
\end{array}\right]
$$

where $\pi_{j}$ is the stationary probability of the Markov chain being in state $j$, exists and can be computed using the relation $\pi=\pi \mathbf{P}$. We find after some calculations that $\pi_{j}=\pi_{0}, j=$ $1, \ldots, t$, and $\pi_{j}=(1-\lambda)^{j-t} \pi_{0}, j=t+1, t+2, \ldots$, and so

$$
\pi_{0}=\frac{1}{t+1 / \lambda}=\frac{\lambda}{\lambda t+1} \text {. }
$$

Hence

$$
\pi_{j}=\left\{\begin{array}{cll}
\frac{\lambda}{\lambda t+1} & , j=0, \ldots, t \\
\frac{(1-\lambda)^{j-t} \lambda}{\lambda t+1}, & j=t+1, t+2, \ldots
\end{array}\right.
$$

We can now derive analytical expressions for the expected energy usage and expected error covariance. For the expected energy usage, since the sensor transmits only when the Markov chain is in states $t, t+1, \ldots$, an energy amount of $E$ is used in reaching the states corresponding to $\bar{P}, f^{t+1}(\bar{P}), f^{t+2}(\bar{P}), \ldots$ Hence

$$
\begin{aligned}
\mathbb{E}[\text { energy }] & =E\left[\pi_{0}+\pi_{t+1}+\pi_{t+2}+\ldots\right] \\
& =E \pi_{0}\left[1+1-\lambda+(1-\lambda)^{2}+\ldots\right] \\
& =\frac{E \pi_{0}}{\lambda}=\frac{E}{\lambda t+1} .
\end{aligned}
$$

For the expected error covariance, we have

$$
\mathbb{E}\left[\operatorname{tr} P_{k \mid k}\right]=\pi_{0} \operatorname{tr}(\bar{P})+\pi_{1} \operatorname{tr}(f(\bar{P}))+\pi_{2} \operatorname{tr}\left(f^{2}(\bar{P})\right)+\ldots
$$

which can be computed numerically. Under the assumption that $\lambda>1-\frac{1}{\|A\|^{2}}, \mathbb{E}\left[\operatorname{tr} P_{k \mid k}\right]$ will be finite, by a similar argument as that used in the proof of Lemma 3.1.

\section{NUMERICAL RESULTS}

We consider an example with parameters

$$
A=\left[\begin{array}{ll}
1.1 & 0.2 \\
0.2 & 0.8
\end{array}\right], C=\left[\begin{array}{ll}
1 & 1
\end{array}\right], Q=I, R=1 .
$$

One can easily compute that

$$
\bar{P}=\left[\begin{array}{rr}
1.3762 & -0.9014 \\
-0.9014 & 1.1867
\end{array}\right] .
$$

The packet reception probability is chosen to be $\lambda=0.8$, and the transmission energy cost $E=1$.

We first consider the finite horizon problem, with $K=5$ and $\beta=0.05$. Fig. 3 and Fig. 4 plots respectively the optimal $\nu_{1}^{*}$ and $\nu_{2}^{*}$ (i.e. $k=1$ and $k=2$ ) for different values of $f^{n}(\bar{P})$, which we recall represents the different values that the error covariance can take. In agreement with Theorem 4.3 , we see a threshold behaviour in the optimal $\nu_{k}^{*}$. In this example we have $P_{0 \mid 0}^{\mathrm{th}}=f^{3}(\bar{P})$ and $P_{1 \mid 1}^{\mathrm{th}}=f^{2}(\bar{P})$, and we see that in general the thresholds are different for different values of $k$.

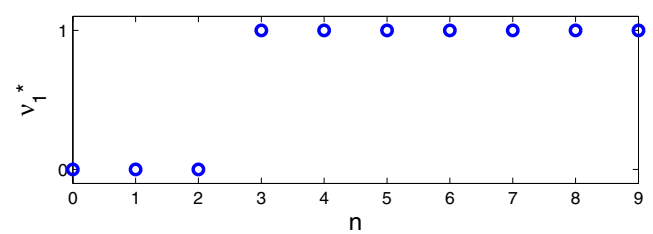

Fig. 3. Finite horizon, $K=5 . \nu_{1}^{*}$ for different values of $f^{n}(\bar{P})$.

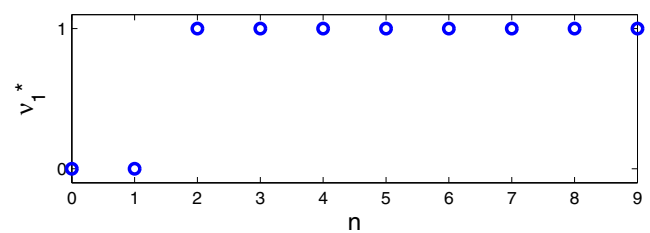

Fig. 4. Finite horizon, $K=5 . \nu_{2}^{*}$ for different values of $f^{n}(\bar{P})$.

We next consider the infinite horizon problem, with $\beta=$ 0.05. Fig. 5 plots the optimal $\nu_{k}^{*}$ for different values of $f^{n}(\bar{P})$, where we again see a threshold behaviour, with $P^{\text {th }}=f^{3}(\bar{P})$. In Fig. 6 we plot the values of the thresholds

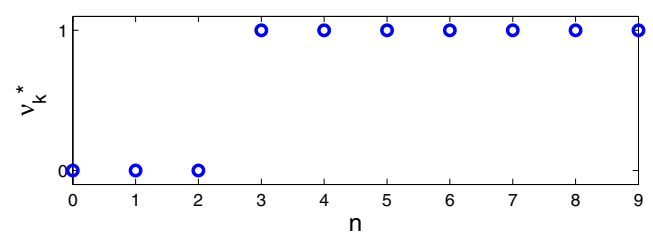

Fig. 5. Infinite horizon. $\nu_{k}^{*}$ for different values of $f^{n}(\bar{P})$.

for different values of $\beta$. As $\beta$ increases, the relative importance of minimizing the error covariance (vs the energy usage) is increased, thus one should transmit more often, leading to decreasing values of the thresholds. Finally, in Fig. 7 we plot the trace of the expected error covariance vs the expected energy, obtained by solving problem (6) for different values of $\beta$, with the values computed using the expressions (12) and (13). Note that the plot is discrete as $t \in \mathbb{N}$ in (12) and (13), see also Fig. 6.

\section{MARKOVIAN PACKET DROPS}

So far in this paper we have considered i.i.d. packet drops. In this section we briefly outline how our results extend to the case where the packet loss process $\left\{\gamma_{k}\right\}$ is a Markov chain, with parameters $p \triangleq \mathbb{P}\left(\gamma_{k}=0 \mid \gamma_{k-1}=1\right)$ and $q \triangleq \mathbb{P}\left(\gamma_{k}=\right.$ $\left.1 \mid \gamma_{k-1}=0\right)$. The probabilities $p$ and $q$ are also known as, respectively, the failure and recovery rates [18]. We shall consider transmission decisions $\nu_{k}\left(P_{k-1 \mid k-1}, \gamma_{k-1}\right)$ 


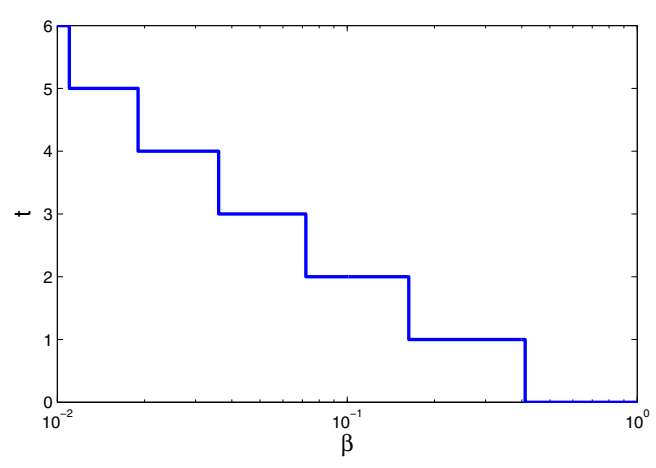

Fig. 6. Infinite horizon. Threshold $P^{\text {th }}$ vs $\beta$, with $f^{t}(\bar{P})=P^{\text {th }}$.

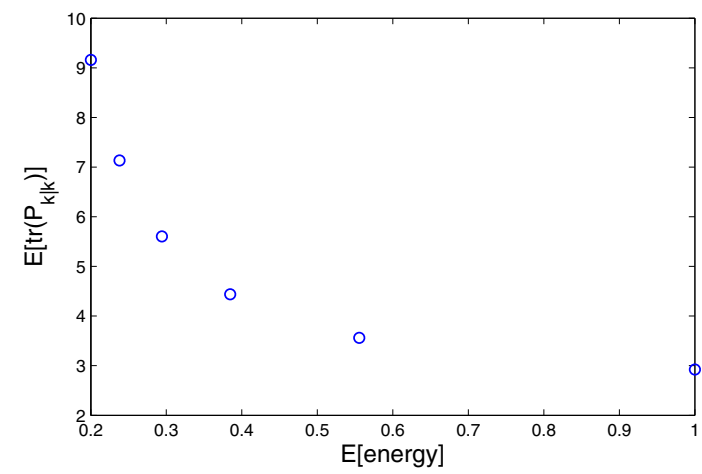

Fig. 7. Infinite horizon. Expected error covariance vs expected energy.

dependent only on $P_{k-1 \mid k-1}$ and $\gamma_{k-1}$, in which case the remote estimator equations will still have the form (1).

The finite horizon problem is:

$$
\min _{\left\{\nu_{k}\right\}} \sum_{k=1}^{K} \mathbb{E}\left[\beta \operatorname{tr} P_{k \mid k}+(1-\beta) \nu_{k} E \mid P_{k-1 \mid k-1}, \gamma_{k-1}, \nu_{k}\right]
$$

for some $\beta \in(0,1)$, where now (c.f. (5))

$$
\begin{aligned}
& \mathbb{E}\left[\operatorname{tr} P_{k \mid k} \mid P_{k-1 \mid k-1}, \gamma_{k-1}, \nu_{k}\right] \\
& =\nu_{k}\left(\gamma_{k-1}(1-p)+\left(1-\gamma_{k-1}\right) q\right) \operatorname{tr} \bar{P} \\
& \quad+\left(1-\nu_{k}\left(\gamma_{k-1}(1-p)+\left(1-\gamma_{k-1}\right) q\right)\right) \operatorname{tr} f\left(P_{k-1 \mid k-1}\right) .
\end{aligned}
$$

The infinite horizon problem is:

$\min _{\left\{\nu_{k}\right\}} \limsup _{K \rightarrow \infty} \frac{1}{K} \sum_{k=1}^{K} \mathbb{E}\left[\beta \operatorname{tr} P_{k \mid k}+(1-\beta) \nu_{k} E \mid P_{k-1 \mid k-1}, \gamma_{k-1}, \nu_{k}\right]$

The following results can be derived:

Lemma 7.1: Let the functions $J_{k}(\cdot, \cdot): \mathcal{S} \times\{0,1\} \rightarrow \mathbb{R}$ be defined recursively for $k=1, \ldots, K$ as:

$$
\begin{aligned}
& J_{K+1}(P, \gamma)=0 \\
& J_{k}(P, \gamma)=\min _{\nu \in\{0,1\}}\{\beta[\nu(\gamma(1-p)+(1-\gamma) q) \operatorname{tr} \bar{P} \\
& \quad+(1-\nu(\gamma(1-p)+(1-\gamma) q)) \operatorname{tr} f(P)] \\
& \quad+(1-\beta) \nu E+\nu(\gamma(1-p)+(1-\gamma) q) J_{k+1}(\bar{P}, 1) \\
& \left.\quad+(1-\nu(\gamma(1-p)+(1-\gamma) q)) J_{k+1}(f(P), 0)\right\} .
\end{aligned}
$$

Then the functions $L_{k}^{1}(\cdot, \cdot): \mathcal{S} \times\{0,1\} \rightarrow \mathbb{R}, k=1, \ldots, K$ and $L_{k}^{0}(\cdot, \cdot): \mathcal{S} \times\{0,1\} \rightarrow \mathbb{R}, k=1, \ldots, K$ given by

$$
\begin{aligned}
L_{k}^{1}(P, \nu) \triangleq & \beta[\nu(1-p) \operatorname{tr} \bar{P}+(1-\nu(1-p)) \operatorname{tr} f(P)] \\
& +(1-\beta) \nu E+\nu(1-p) J_{k+1}(\bar{P}, 1) \\
& +(1-\nu(1-p)) J_{k+1}(f(P), 0) \\
L_{k}^{0}(P, \nu) \triangleq & \beta[\nu q \operatorname{tr} \bar{P}+(1-\nu q) \operatorname{tr}(f(P))] \\
& +(1-\beta) \nu E+\nu q J_{k+1}(\bar{P}, 1) \\
& +(1-\nu q) J_{k+1}(f(P), 0)
\end{aligned}
$$

are submodular on $\mathcal{S} \times\{0,1\}$.

\section{Proof: Define}

$$
\begin{aligned}
& F^{1}(P, \nu) \triangleq \beta[\nu(1-p) \operatorname{tr} \bar{P}+(1-\nu(1-p)) \operatorname{tr} f(P)]+(1-\beta) \nu E \\
& G_{k}^{1}(P, \nu) \triangleq \nu(1-p) J_{k+1}(\bar{P}, 1)+(1-\nu(1-p)) J_{k+1}(f(P), 0) .
\end{aligned}
$$

Using similar arguments as in the proof of Lemma 4.2, we can show that $F^{1}(.,$.$) and G_{k}^{1}(.,$.$) are submodular on \mathcal{S} \times$ $\{0,1\}$, which then implies that

$$
L_{k}^{1}(P, \nu)=F^{1}(P, \nu)+G_{k}^{1}(P, \nu)
$$

is submodular. The submodularity of $L_{k}^{0}(.,$.$) can be shown$ in the same way.

Lemma 7.1 implies that in the finite horizon problem (14), for each $k$ there exist two (in general different) thresholds $P_{k-1 \mid k-1}^{t h, 1}$ and $P_{k-1 \mid k-1}^{t h, 0} \in \mathcal{S}, k=1, \ldots, K$, such that when $\gamma_{k-1}=1$ then $\nu_{k}^{*}=0$ if and only if $P_{k-1 \mid k-1}<P_{k-1 \mid k-1}^{t h, 1}$; and when $\gamma_{k-1}=0$ then $\nu_{k}^{*}=0$ if and only if $P_{k-1 \mid k-1}<$ $P_{k-1 \mid k-1}^{t h, 0}$.

For the infinite horizon problem (15), similar to the proof of Theorem 4.3 (ii), the optimal policy will be such that when $\gamma_{k-1}=1$ then $\nu_{k}^{*}=0$ if and only if $P_{k-1 \mid k-1}<P^{t h, 1}$; and when $\gamma_{k-1}=0$ then $\nu_{k}^{*}=0$ if and only if $P_{k-1 \mid k-1}<P^{t h, 0}$, for some constant thresholds $P^{t h, 1}$ and $P^{t h, 0} \in \mathcal{S}$.

\section{CONCLUSION}

This paper has shown that in event triggered estimation with packet drops, a threshold policy is optimal in the sense that it will minimize a convex combination of the expected error covariance and expected energy usage. Future work includes the study of triggering policies with imperfect feedback acknowledgements, and deriving structural properties of the optimal scheduling policy for systems with multiple sensors.

\section{APPENDIX}

\section{Proof of Lemma 3.1}

We will verify the conditions $\left(\mathrm{CAV}^{*} 1\right)$ and $\left(\mathrm{CAV}^{*} 2\right)$ given in Corollary 7.5.10 of [14], which guarantee the existence of solutions to the Bellman equation for average cost problems with countably infinite state space. Condition $\left(\mathrm{CAV}^{*} 1\right)$ says that there exists a standard policy $d$ such that the recurrent class $R_{d}$ of the Markov chain induced by $d$ is equal to the whole state space $S$, where $d$ is a standard policy if there exists a state $z$ such that the expected first passage time $\tau_{i, z}$ from $i$ to $z$ satisfies $\tau_{i, z}<\infty, \forall i \in S$, and the expected first passage cost $c_{i, z}$ from $i$ to $z$ satisfies $c_{i, z}<\infty, \forall i \in S$. 
Condition $\left(\mathrm{CAV}^{* 2}\right)$ says that given $U>0$, the set $D_{U}=$ $\{i \in S \mid c(i, a) \leq U$ for some $a\}$ is finite, where $c(i, a)$ is the cost at each stage when in state $i$ and using action $a$.

To verify $\left(\mathrm{CAV}^{*} 1\right)$, let $d$ be the policy that always transmits, i.e. $\nu_{k}=1, \forall k$. Let state $i$ of the induced Markov chain correspond to the value $f^{i}(\bar{P}), i=0,1,2, \ldots$, where we define $f^{0}(\bar{P}) \triangleq \bar{P}$. The state diagram of the induced Markov chain is given in Fig 8 , with state space $S=\{0,1,2, \ldots\}$.

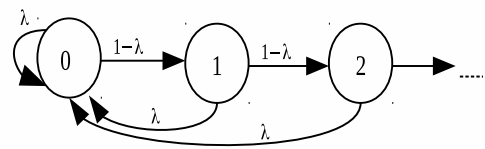

Fig. 8. Markov chain for policy of always transmitting

Let $z=0$. Then the expected first passage time from state $i$ to state $z=0$ is

$$
\tau_{i, z}=\lambda+2(1-\lambda) \lambda+3(1-\lambda)^{2} \lambda+\cdots=\frac{1}{\lambda}<\infty .
$$

The expected cost of a first passage from state $i$ to state $z=0$ is

$$
\begin{aligned}
& c_{i, z}=\beta \operatorname{tr} f^{i}(\bar{P})+(1-\beta) E+(1-\lambda) c_{(i+1) 0} \\
& =\beta \operatorname{tr} f^{i}(\bar{P})+(1-\beta) E+(1-\lambda)\left(\beta \operatorname{tr} f^{i+1}(\bar{P})\right. \\
& \quad+(1-\beta) E)+(1-\lambda)^{2}\left(\beta \operatorname{tr} f^{i+2}(\bar{P})+(1-\beta) E\right)+\ldots \\
& =\beta \sum_{n=0}^{\infty}(1-\lambda)^{n} \operatorname{tr} f^{i+n}(\bar{P})+\frac{(1-\beta) E}{\lambda} .
\end{aligned}
$$

We have

$$
\begin{aligned}
\frac{(1-\lambda)^{n+1} \operatorname{tr} f^{i+n+1}(\bar{P})}{(1-\lambda)^{n} \operatorname{tr} f^{i+n}(\bar{P})} & =(1-\lambda) \frac{\operatorname{tr}\left(A f^{i+n}(\bar{P}) A^{T}+Q\right)}{\operatorname{tr} f^{i+n}(\bar{P})} \\
& \leq(1-\lambda)\left(\|A\|^{2}+\frac{\operatorname{tr} Q}{\operatorname{tr} f^{i+n}(\bar{P})}\right)
\end{aligned}
$$

where the inequality uses Fact 8.12 .28 of [19]. Since $A$ is unstable, $\operatorname{tr} f^{i+n}(\bar{P}) \rightarrow \infty$ as $n \rightarrow \infty$, and so

$$
\lim _{n \rightarrow \infty} \frac{(1-\lambda)^{n+1} \operatorname{tr} f^{i+n+1}(\bar{P})}{(1-\lambda)^{n} \operatorname{tr} f^{i+n}(\bar{P})} \leq(1-\lambda)\|A\|^{2} .
$$

Then by the ratio test, the condition $(1-\lambda)\|A\|^{2}<1$, or equivalently $\lambda>1-\frac{1}{\|A\|^{2}}$, is sufficient for the infinite series in the last line of (16) to converge. Hence $d$ is a standard policy. Furthermore, one can see from Fig. 8 that the positive recurrent class $R_{d}$ of the induced Markov chain is equal to $S$, which verifies $\left(\mathrm{CAV}^{*} 1\right)$.

Since the cost per stage $c(i, a)$ corresponds to $\beta \operatorname{tr} P_{k \mid k}+$ $(1-\beta) \nu_{k} E$, condition $\left(\mathrm{CAV}^{*} 2\right)$ can be easily verified.

\section{REFERENCES}

[1] Y. Xu and J. P. Hespanha, "Optimal communication logic in networked control systems," in Proc. CDC, Paradise Islands, Bahamas, Dec. 2004, pp. 842-847.

[2] L. Li, M. Lemmon, and X. Wang, "Event-triggered state estimation in vector linear processes," in Proc. ACC, Baltimore, MD, Jun. 2010, pp. 2138-2143.
[3] S. Trimpe and R. D'Andrea, "Event-based state estimation with variance-based triggering," in Proc. IEEE CDC, Maui, Hawaii, Dec. 2012, pp. 6583-6590.

[4] M. Xia, V. Gupta, and P. J. Antsaklis, "Networked state estimation over a shared communication medium," in Proc. ACC, Washington, DC, Jun. 2013, pp. 4134-4319.

[5] J. Wu, Q.-S. Jia, K. H. Johansson, and L. Shi, "Event-based sensor data scheduling: Trade-off between communication rate and estimation quality," IEEE Trans. Autom. Control, vol. 58, no. 4, pp. 1041-1046, Apr. 2013.

[6] D. Han, Y. Mo, J. Wu, B. Sinopoli, and L. Shi, "Stochastic eventtriggered sensor scheduling for remote state estimation," in Proc. CDC, Florence, Italy, Dec. 2014, pp. 6079-6084.

[7] P. Tabuada, "Event-triggered real-time scheduling of stabilizing control tasks," IEEE Trans. Autom. Control, vol. 52, no. 9, pp. 1680-1685, Sep. 2007.

[8] M. Rabi and K. H. Johansson, "Scheduling packets for event-triggered control," in Proc. Europ. Contr. Conf., Budapest, Hungary, Aug. 2009, pp. 3779-3784.

[9] C. Ramesh, H. Sandberg, and K. H. Johansson, "Design of statebased schedulers for a network of control loops," IEEE Trans. Autom. Control, vol. 58, no. 8, pp. 1962-1975, Aug. 2012.

[10] D. E. Quevedo, V. Gupta, W.-J. Ma, and S. Yüksel, "Stochastic stability of event-triggered anytime control," IEEE Trans. Autom. Control, vol. 59, no. 12, pp. 3373-3379, Dec. 2014.

[11] B. Sinopoli, L. Schenato, M. Franceschetti, K. Poolla, M. I. Jordan, and S. S. Sastry, "Kalman filtering with intermittent observations," IEEE Trans. Autom. Control, vol. 49, no. 9, pp. 1453-1464, September 2004.

[12] L. Shi, M. Epstein, and R. M. Murray, "Kalman filtering over a packetdropping network: A probabilistic perspective," IEEE Trans. Autom. Control, vol. 55, no. 3, pp. 594-604, Mar. 2010.

[13] D. P. Bertsekas, Dynamic Programming and Optimal Control, Volume I, 2nd ed. Massachusetts: Athena Scientific, 2000.

[14] L. I. Sennott, Stochastic Dynamic Programming and the Control of Queueing Systems. New York: Wiley-Interscience, 1999.

[15] D. M. Topkis, "Minimizing a submodular function on a lattice," Operations Research, vol. 26, no. 2, pp. 305-321, Mar-Apr 1978.

[16] —, Supermodularity and Complementarity. New Jersey: Princeton University Press, 1998.

[17] M. H. Ngo and V. Krishnamurthy, "Optimality of threshold policies for transmission scheduling in correlated fading channels," IEEE Trans. Commun., vol. 57, no. 8, pp. 2474-2483, Aug. 2009.

[18] M. Huang and S. Dey, "Stability of Kalman filtering with Markovian packet losses," Automatica, vol. 43, pp. 598-607, 2007.

[19] D. S. Bernstein, Matrix Mathematics, 2nd ed. New Jersey: Princeton University Press, 2009. 